\title{
Deep Learning-Based Automatic Detection of Rectal Polyps Using Abdominal CT Images Guided by Cold Snare Polypectomy
}

\author{
Haijun Lin $\mathbb{D}^{1},{ }^{1}$ Qi Chen $\left(\mathbb{D},{ }^{2}\right.$ Caijuan Li $\mathbb{D},{ }^{3}$ Aifen Zheng $\mathbb{D},{ }^{3}$ Lei Yang $\mathbb{D},{ }^{4}$ Jiemin Hong $\mathbb{D}^{1},{ }^{1}$ \\ Hanqing Chen $\mathbb{D},{ }^{1}$ Xuni He $\mathbb{D}^{1},{ }^{1}$ and Wuna Feng $\mathbb{D}^{4}$ \\ ${ }^{1}$ Department of Gastroenterology, Ningbo Yinzhou No. 2 Hospital, Yinzhou, Ningbo 315100, China \\ ${ }^{2}$ Department of Infectious Diseases, Ningbo Yinzhou No. 2 Hospital, Yinzhou, Ningbo 315100, China \\ ${ }^{3}$ Endoscopy Center, Ningbo Yinzhou No. 2 Hospital, Yinzhou, Ningbo 315100, China \\ ${ }^{4}$ Emergency Department, Ningbo Yinzhou No. 2 Hospital, Yinzhou, Ningbo 315100, China
}

Correspondence should be addressed to Wuna Feng; wunafeng@yzpc.edu.cn

Received 20 May 2021; Revised 20 June 2021; Accepted 28 June 2021; Published 5 July 2021

Academic Editor: Gustavo Ramirez

Copyright $(92021$ Haijun Lin et al. This is an open access article distributed under the Creative Commons Attribution License, which permits unrestricted use, distribution, and reproduction in any medium, provided the original work is properly cited.

The study drew attention to the therapeutic effects of cold snare polypectomy guided by a deep convolutional neural network(CNN-) based abdominal CT and hot snare polypectomy (HSP) on colonic and rectal polyps. Specifically, 90 patients were enrolled into a blank group, a control (Ctrl) group, and an experimental group. The blank group accepted HSP, the Ctrl accepted cold snare polypectomy, and the experimental group accepted cold snare polypectomy guided by deep CNN-based CT images. It was found that the experimental group had the lowest false-positive rate (9.2\%) in polyp detection in contrast with the Ctrl (21.4\%) and the blank group $(52.3 \%)(P<0.05)$. The complete resection rate of large polyps in the experimental group was the highest $(P<0.05)$, and its operation time $(2.91 \pm 0.75 \mathrm{~min})$ was obviously shorter versus the blank group $(6.18 \pm 1.19 \mathrm{~min})(P<0.05)$. In conclusion, the cold snare polypectomy under the guidance of deep CNN-based CT has a relatively high complete resection rate and detection accuracy of polyps with a low complication rate, which can be adopted clinically.

\section{Introduction}

The colonic and rectal polyp is a kind of disease that frequently occurs in people over the age of 40 and is manifested as a protrusion of the mucosal surface of the colon and rectum or a bulge of the intestinal cavity. Before its pathological properties are determined, it is called a polyp, that is, a "pump" that grows in the intestine. The incidence rate increases with age and that in men is higher than that in women. Polyps can be divided into adenomatous polyps and nonadenomatous polyps according to their pathological types. Nonadenomatous polyps include hyperplastic polyps, inflammatory polyps, and hamartomas. More than $80 \%$ of colorectal cancers are induced by adenomatous polyps. Therefore, adenomatous polyps are precancerous lesions of colorectal cancer. Colonic and rectal polyps are mostly caused by genetic factors or Helicobacter pylori infection. They are polygenic genetic diseases caused by mutations in two or more pairs of disease-causing genes and are jointly affected by genetic factors and environmental factors. The commonly used treatment methods for this disease are colonoscopy, primary treatment (such as chronic ulcerative colitis and Crohn's granuloma), and surgical treatment such as removing colonic and rectal polyps by HSP (applicable to the diameter of 6 to $9 \mathrm{~mm}$ raised or pedicled polyps). The snare is one of the commonly used digestive endoscopy accessories in endoscopic treatment. It is mainly used for the resection of gastrointestinal polyps, raised lesions, flat lesions, and early gastrointestinal cancer, such as endoscopic mucosal resection (EMR) and multiband mucosectomy (MBM). The use of the cold snare technique to treat colorectal polyps can obviously reduce intraoperative blood loss, which is able to monitor intraoperative bleeding caused by submucosal vascular injury in real-time and demonstrates better treatment effects on colorectal polyps with relatively high safety and effectiveness. 
In recent years, computer technology has been extensively used in medical diagnosis, such as the commonly used Computed Tomography (CT) and Magnetic Resonance Imaging (MRI) $[1,2]$. CT is an X-ray computer tomography technology. Because different tissues can absorb different amounts of X-rays, the internal structure of the human body can be displayed in the form of three-dimensional imaging using this technology, which provides convenience and basis for clinical diagnosis $[3,4]$. $\mathrm{CNN}$ is a feedforward neural network, which can effectively reduce the complexity of feedback neural network $[5,6]$ and can be used to identify some distorted but not deformed two-dimensional images. It is one of the representative algorithms of deep learning [7]. The wide application of CNN in medical image classification provides convenience for research. Therefore, neural network recognition technology has attracted attention from all walks of life and is widely used in image segmentation [8]. The neural network has a huge number of connections, is easy to introduce spatial information, and can better solve the problems of unevenness and noise in image recognition. The first $\mathrm{CNN}$ is a time delay network proposed by foreign scholars and experts [9], which is used for speech recognition, and the second is a translational invariant artificial neural network, which is used for medical image detection and recognition [10-14]. Then, with the introduction of deep learning theory and the updating of numerical computing equipment, CNNs have progressed gradually, and its representation learning capabilities begin to receive widespread attention.

In the study, 90 patients were enrolled into a blank group, a Ctrl group, and an experimental group for HSP, cold snare polypectomy, and the cold snare polypectomy guided by deep CNN-based CT images, respectively, so as to compare the therapeutic effects of cold snare polypectomy guided by deep CNN-based abdominal CT and HSP on colonic and rectal polyps.

\section{Material and Methods}

2.1. The Basic Data. Ninety patients with colonic and rectal polyps in our hospital between September 2018 to September 2020 were selected as research subjects. There were 48 males and 42 females (age range 40-60), and they were enrolled into a blank group (treated by HSP), a Ctrl (treated by cold snare polypectomy), and an experimental group (treated by cold snare polypectomy under the guidance of deep CNNbased CT images) in random. The study was approved by the medical ethics committee, and the patients and their families have been informed of the study and signed the consent forms.

Inclusion criteria were as follows: (i) patients between 40 and 60 years old; (ii) patients with clear consciousness and who can cooperate with treatment and sample collection; (iii) patients with colonic and rectal polyps confirmed by premenstrual examination; (iv) patients with complete clinical data and information; (v) patients who had no history of mental illness and were emotionally stable.

Exclusion criteria were as follows: (i) patients who withdrew and transferred for treatment due to personal reasons; (ii) patients with other serious diseases or infectious diseases; (iii) patients with incomplete case data; (iv) patients with severe colonic and rectal diseases or patients who had accepted similar treatments for this disease; (v) patients with other system or organ diseases; (vi) patients who did not cooperate with the doctors due to personal or other factors.

2.2. Preoperative Preparation and CT Scan. The three groups of patients should have low-fiber food before the abdominal CT examination and started fasting 12 hours before the examination. Eight hours before the examination, they should take 1L polyethylene glycol electrolyte solution to clean the intestines. The scan voltage and current were set to $120 \mathrm{KV}$ and $120 \mathrm{~mA}$, respectively, scan layer thickness $0.5 \mathrm{~mm}$, and pitch factor 0.828 . The patient was in the left decubitus position, and it should be ensured that enough air is injected into the intestine to expand the intestine. Then, the entire colon and rectum were scanned in the sequence from supine position to prone position. After the scan, the data were uploaded to the workstation, and the scanned images were reviewed and diagnosed by physicians with about ten years of experience in intestinal CT diagnosis.

2.3. Deep CNN Learning Algorithm. In the study, the experimental group accepted cold snare polypectomy guided by deep CNN-based CT images. In the segmentation of CT images, the parameters are automatically adjusted by supervised learning so that the network can learn characteristics automatically. The image processing is shown in Figure 1.

In general, the $\mathrm{CNN}$ algorithm mainly refers to a linear operation method for grid data, including several basic simple operation processes. Compared with other neural networks, CNN has independent connection and calculation methods, but they are all based on the backpropagation algorithm, and the network parameters are adjusted and optimized on this basis.

$$
T(n)=\int_{-\infty}^{+\infty} I(x) O(n-x) Y_{x},
$$

where $I(x)$ and $O(x)$ are integral functions, $I(x)$ represents the input, $O(x)$ represents the convolutional kernel, and $T(n)$ is the feature mapping, expressed as follows:

$$
T(n)=\sum_{x=-\infty}^{+\infty} I(x) O(n-x) .
$$

In practice, for discrete data in the image, $I(x)$ and $O(x)$ are both discrete variables. Then, the convolution of $I(x)$ and $O(x)$ is expressed as equation (2). CNN can recognize images by sliding window, selective search, and other methods. First, it can judge whether the window is the target and defines it as the regression problem of the occurrence probability of each target in image segmentation. This method uses full CNN deep learning-based CT images to perform colonic and rectal polyps image segmentation, and the mean intersection over Union (mioU) is taken as the final judgment index. 


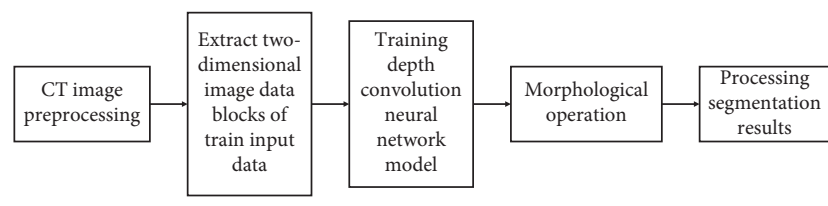

FIGURE 1: The processing of deep CNN-based CT images.

$$
R=\frac{|M \cap N|}{|M \cup N|},
$$

where $R$ represents the mioU, $M$ represents the point set satisfying $Y_{i, j}=1$, and $N$ represents the point set satisfying $Y_{i, \mathrm{j}}>0.5$. In segmentation of colonic and rectal polyp images, the sensitivity is always used to evaluate the detection rate, calculated as follows:

$$
X=\frac{Y p}{(Y p+N q)},
$$

where $Y p$ represents the positive colonic and rectal polyps detected and $\mathrm{Nq}$ represents the negative colonic and rectal polyps detected.

2.4. Evaluation Indicators. After three methods were adopted to remove colonic and rectal polyps, the treatment time, detection accuracy, and complication rate of the three methods were compared to evaluate their therapeutic effect.

2.5. Statistical Methods. The data were processed by SPSS19.0 version statistical software, mean \pm standard deviation $(\bar{x}+s)$ illustrated how to calculate the measurement data, and the counting data were expressed in percentage (\%). Paired $t$-test was applied to compare the treatment time between groups, and analysis of variance was adopted to compare the polypectomy effect. The difference was statistically significant with $P<0.05$.

\section{Results}

3.1. CT Images of Lesions in Patients with Colorectal Cancer. Figure 2 is a CT image of a patient with colon polyps. It was evident that there were multiple round barium smears of varying sizes in the sigmoid colon, with clear edges, and the colon and sigmoid colon were inflated well. There was a filling defect on the inner side of the descending colon, with obviously narrowed bowel lumen. The mucosa was obviously damaged, niches were visible, and the bowel cavity was obviously narrowed. The polyp was not smooth with an irregular shape. The top of the pedicled polyp was enlarged and entered the pedicle, which shortened the pedicle. A mass with a broad base was formed, and there was a concave notch on the intestinal wall at the base of the polyp. The polyp was relatively large and the base was wide, and there were many small filling defects that were diffusely distributed, and the mucosal folds were obviously disordered and deformed.

Figure 3 is a CT image of a patient with rectal polyps. There were irregular filling defects in the rectum. The intestinal tube showed eccentric stenosis that was lobulated

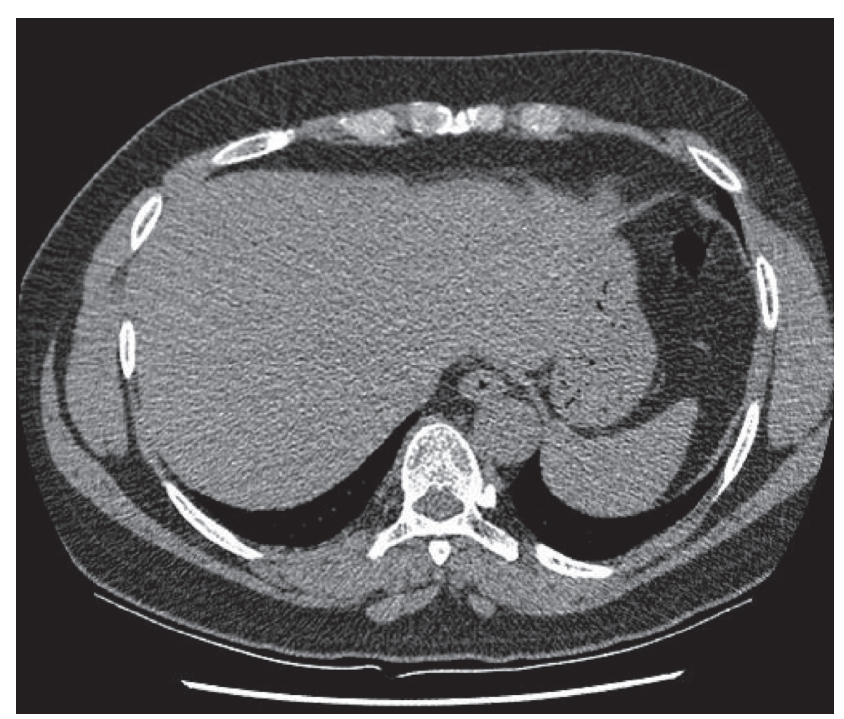

Figure 2: The CT image of a colonic polyp.

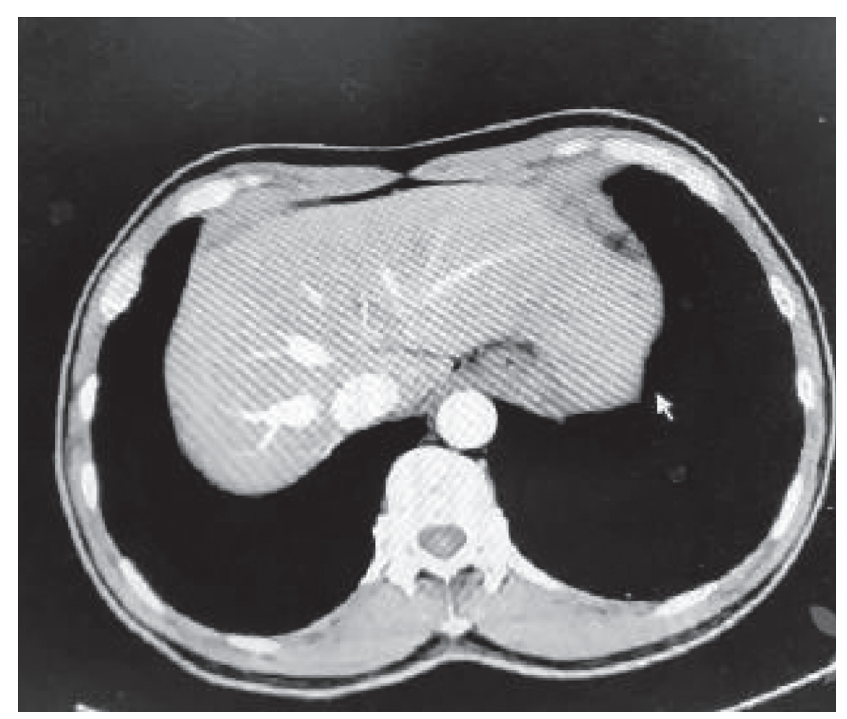

FIgURE 3: The CT image of a rectal polyp.

and irregular, and the mucosal folds were disordered. The rectal wall was unevenly thickened, and there were soft tissue shadows in the rectal cavity. After an enhanced scan, the image was moderately enhanced. The surrounding fat clearance was not clear, and swollen lymph nodes can be observed behind the rectum. The mucosa surface of the thickened intestinal wall was mostly uneven, the boundary with the normal intestinal wall was clear, and there were small ulcers that looked like volcanic craters. The intestinal cavity was narrow, and the mucosa of the narrowed segment appeared jagged.

3.2. Types and Locations of Polyps. Figure 4 shows the types and locations of polyps of 90 patients. The lesions in the three groups were mainly located in the sigmoid colon, ascending colon, descending colon, rectum, and transverse 


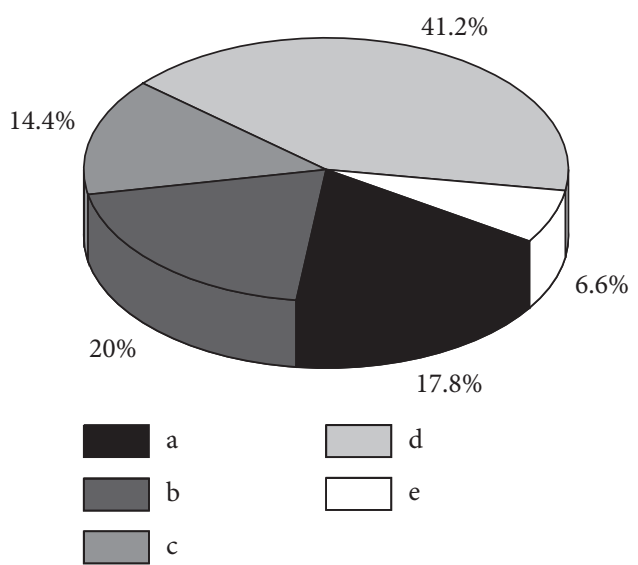

FIgURE 4: The types and locations of polyps. a: descending colon polyps; b: ascending colon polyps; c: transverse colon polyps; $d$ : sigmoid polyps; e: rectal polyps.

colon. The incidence of sigmoid polyps was highest (41.2\%), followed by ascending colon and descending colon $(20 \%$ and $17.8 \%$, respectively) and transverse colon and rectal polyps (6.6\% and $14.4 \%$, respectively).

Figure 5 shows the types of colonic and rectal polyps and the number of patients with each kind of polyps. The large polyp was no less than $1 \mathrm{~cm}$, and the moderate polyp was between 5 and $9 \mathrm{~mm}$. The numbers of patients with large and moderate colonic and rectal polyps was all 16-18 and 12-14 in the three groups $(P>0.05)$.

3.3. The Comparison of Detection Accuracy for Polyps. Figure 6 presents the comparison of false-positive rate in detection of colonic and rectal polyps. That in blank group, Ctrl, and experimental groups was 52.3\%, 21.4\%, and 9.2\%, respectively $(P<0.05)$.

Figure 7 presents the comparison of false-negative rate in colonic and rectal polyp detection. That in the blank group, Ctrl, and experimental group was $45.8 \%, 16.2 \%$, and $6.4 \%$, respectively $(P<0.05)$.

3.4. Comparison of the Complete Resection Rate of Polyps. Figure 8 shows the complete resection rates of moderate polyps in the three groups. The complete resection rates of the control and experimental groups were $76.9 \%$ and $97.3 \%$, respectively. The experimental group had the highest complete resection rate among the three groups $(P<0.05)$, whereas the other two groups showed no notable difference $(P>0.05)$.

Figure 9 shows the complete resection rates of large polyps in the three groups, which were $71 \%$ and $96.8 \%$ in the Ctrl and the experimental group, respectively. In contrast with the blank group, the complete resection rates of large polyps in the other two groups were higher $(P<0.05)$, and that of the experimental group was obviously higher versus the Ctrl $(P<0.05)$.

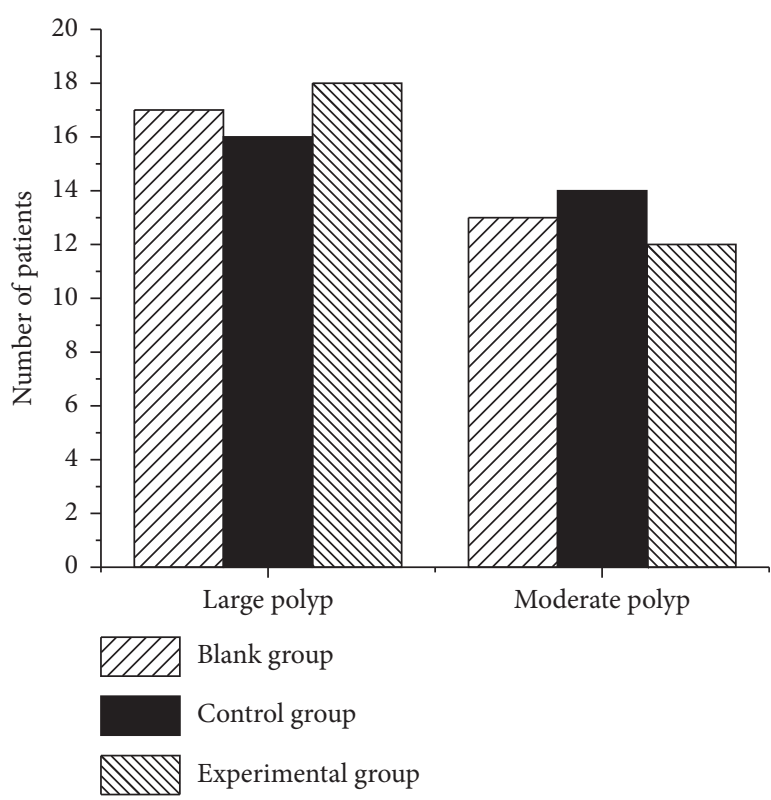

FIGURE 5: The types of colonic and rectal polyps.

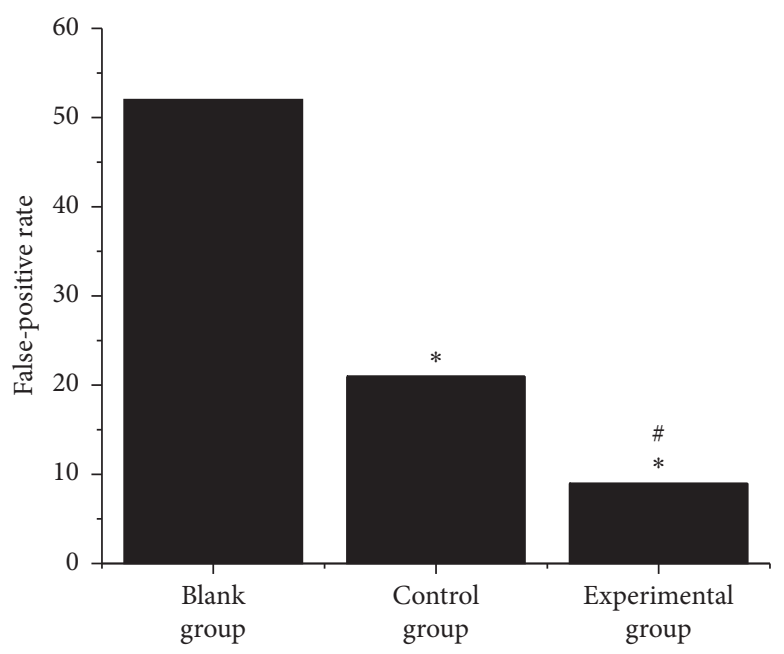

FIgURE 6: The false-positive rates in polyp detection. * indicates that there was a notable difference in contrast with the blank group $(P<0.05)$; \# indicates that there was a notable difference in contrast with the Ctrl $(P<0.05)$ (with the same meaning below).

3.5. The Postoperative Complication Rate. Figure 10 shows results for postoperative complication rate. The main postoperative complications were hemorrhage, abdominal pain, and abdominal distension. The incidence of abdominal distension was the highest, accounting for $52.5 \%$, followed by abdominal pain and hemorrhage, which accounted for $30 \%$ and $17.5 \%$, respectively.

Figure 11 shows the number of patients with hemorrhage, abdominal pain, and abdominal distension in each group after surgery. It was evident that the incidence of abdominal pain and abdominal distension was high in each 


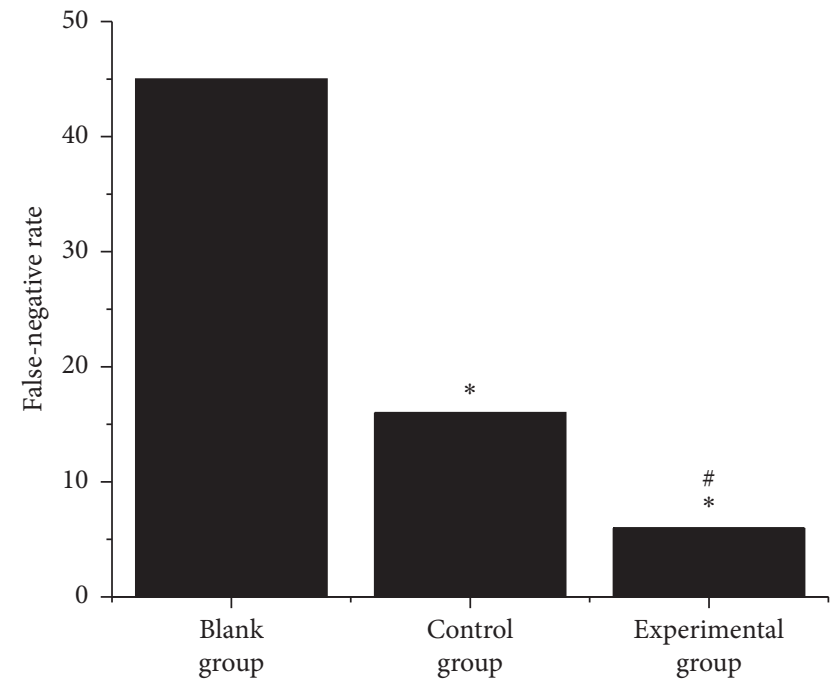

Figure 7: The false-negative rate in polyp detection.

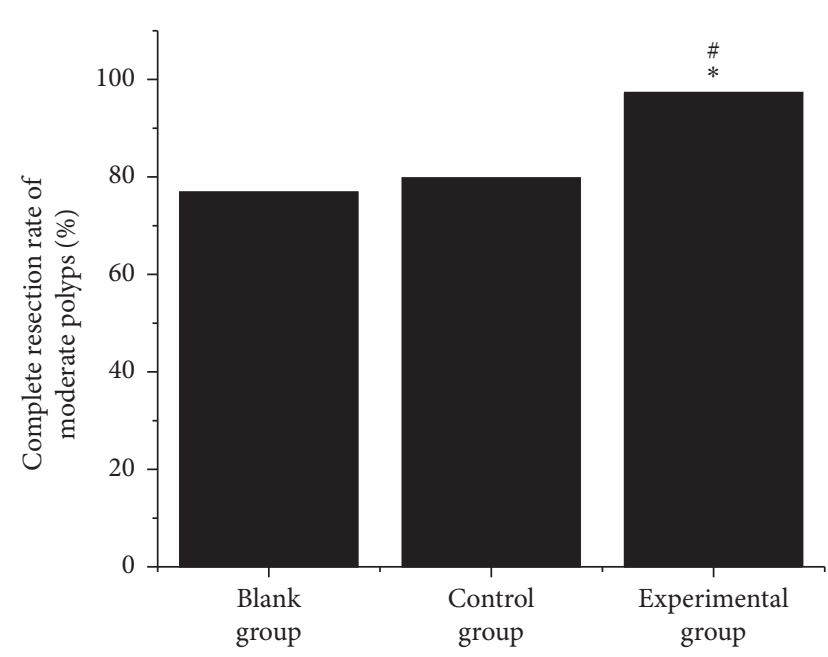

Figure 8: The complete resection rates for moderate polyps.

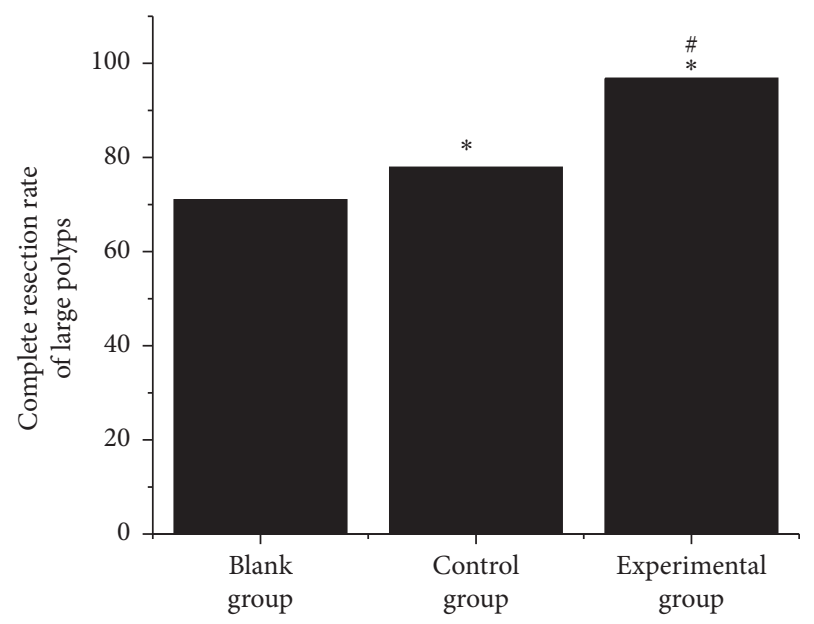

Figure 9: The complete resection rate for large polyps.

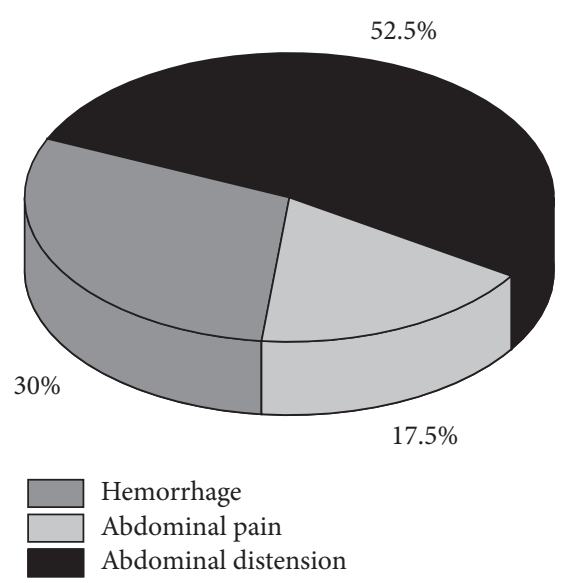

FIgURE 10: Types and occurrence of complications.
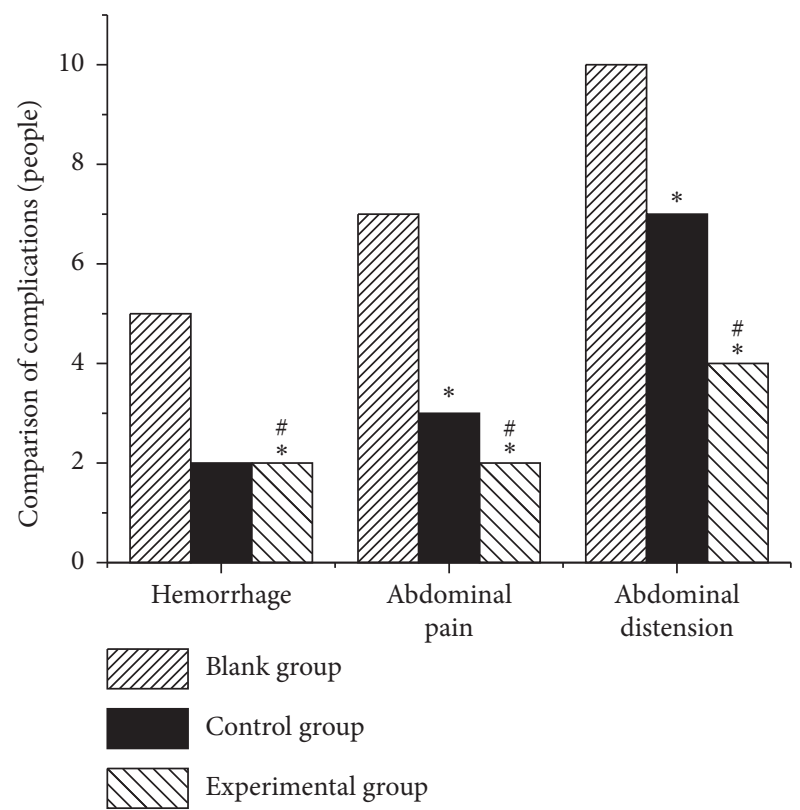

FIGURE 11: Types and incidence of complications.

group of patients. The incidence of abdominal pain in the experimental group was the lowest, and that in the Ctrl was lower than the blank group $(P<0.05)$. The incidence of hemorrhage was low in both the three groups and that was the highest in the blank group $(P<0.05)$, whereas there was no notable difference between the other two groups $(P>0.05)$.

3.6. Time for Surgical Removal of Polyps. Figure 12 shows the comparison results of resection time. It was evident that the resection times of the blank group, control group, and experimental group were $6.18 \pm 1.19 \mathrm{~min}, 4.02 \pm 0.89 \mathrm{~min}$, and $2.91 \pm 0.75 \mathrm{~min}$, respectively $(P<0.05)$. 


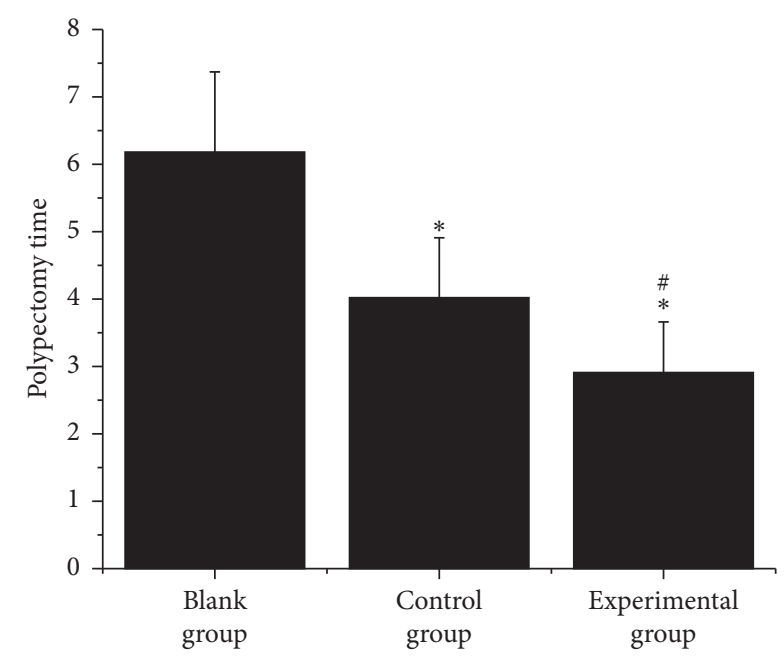

Figure 12: Comparison of the resection time of polyps.

\section{Discussion}

Studies have found that the rate of visits for colonic and rectal polyps in China has been low, which leaves hidden dangers for the health of the digestive system. Polyps can grow throughout the digestive tract, including the colon and rectum $[15,16]$. There are few small intestinal polyps, and they are called colon polyps until their pathological properties are determined [17]. The incidence of colonic and rectal polyps will increase with age, and males are more prone to develop this disease. It is caused by many factors such as age, diet, lifestyle, inflammatory stimulation, genes and inheritance, intestinal mucosal damage, and intestinal inflammation, but it is generally believed that the formation of polyps is closely related to genetic mutations. Juvenile polyps are mostly hamartoma, which may be related to abnormal embryonic development. If diagnosis and treatment are not performed in time, it is easy to induce diseases such as colon cancer [18]. Therefore, early diagnosis and treatment of colonic and rectal cancer are crucial clinically. Related foreign research has found that $80 \%$ of $7-9 \mathrm{~mm}$ polyps are treated with snare polypectomy. For elevated type Ip, Isp, and Is diseases, the Chinese Medical Association Guidance also recommends snare polypectomy treatment [19].

In the study, 90 patients were enrolled into a blank group, a Ctrl group, and an experimental group. The blank group accepted HSP, the Ctrl accepted cold snare polypectomy, and the experimental group accepted cold snare polypectomy guided by deep CNN-based CT images. Further, the therapeutic effect of the three groups was compared. It was found that the experimental group had the lowest false-positive rate $(9.2 \%)$ in polyp detection in contrast with the Ctrl (21.4\%) and the blank group (52.3\%) $(P<0.05)$. The complete resection rate of large polyps in the experimental group was the highest $(P<0.05)$, and its operation time $(2.91 \pm 0.75 \mathrm{~min})$ was obviously shorter versus the blank group $(6.18 \pm 1.19 \mathrm{~min})(P<0.05)$. This was in line with the research results of Din et al. [20], indicating that the cold snare polypectomy under the guidance of deep CNN-based CT can be adopted clinically.

\section{Conclusion}

In the study, 90 patients with colonic and rectal polyps were enrolled into a blank group, a Ctrl group, and an experimental group. The blank group accepted HSP, the Ctrl accepted cold snare polypectomy, and the experimental group accepted cold snare polypectomy guided by deep CNN-based CT images. Further, their therapeutic effect was compared. It was found that the cold snare polypectomy under the guidance of deep CNN-based CT had a relatively high complete resection rate and detection accuracy of polyps with a low complication rate, which can be adopted clinically. However, the sample size is small in the study, which may cause deviations in results. In follow-up studies, the sample size should be expanded to explore further the influence of deep CNN learning algorithm on CT analysis of colonic and rectal polyps. The study provides data support for the clinical diagnosis and treatment of rectal polyps.

\section{Data Availability}

No data were used to support this study.

\section{Conflicts of Interest}

The authors declare that they have no conflicts of interest.

\section{References}

[1] P. J. Pickhardt, B. D. Pooler, D. H. Kim, C. Hassan, K. A. Matkowskyj, and R. B. Halberg, "The natural history of colorectal polyps," Gastroenterology Clinics of North America, vol. 47, no. 3, pp. 515-536, 2018.

[2] L. Herszényi, "The "difficult" colorectal polyps and adenomas: practical aspects," Digestive Diseases, vol. 37, no. 5, pp. 394-399, 2019.

[3] M. B. Loughrey and N. A. Shepherd, "Problematic colorectal polyps," Surgical Pathology Clinics, vol. 10, no. 4, pp. 947-960, 2017.

[4] S. Tanaka, Y. Saitoh, T. Matsuda, M. Igarashi, T. Matsumoto, Y. Iwao et al., "Evidence-based clinical practice guidelines for management of colorectal polyps," Journal of Gastroenterology, vol. 50, no. 3, pp. 252-260, 2015.

[5] L. Jiang and Y. F. Jiao, "Clinicopathological features of nonneoplastic colorectal polyps," Zhonghua Bing Li Xue Za Zhi, vol. 48, no. 2, pp. 98-101, 2019.

[6] K. Okamoto, S. Kitamura, T. Kimura, T. Nakagawa, M. Sogabe, H. Miyamoto et al., "Clinicopathological characteristics of serrated polyps as precursors to colorectal cancer: current status and management," Journal of Gastroenterology and Hepatology, vol. 32, no. 2, pp. 358-367, 2017.

[7] A. K. Safayeva and N. Y. Bayramov, "The importance of colonoscopy in the treatment of colorectal polyps and colorectal cancer screening," Annali Italiani di Chirurgia, vol. 90, pp. 311-317, 2019.

[8] W. Zhao, J. Yang, Y. Sun et al., "3D deep learning from CT scans predicts tumor invasiveness of subcentimeter pulmonary adenocarcinomas," Cancer Research, vol. 78, no. 24, pp. 6881-6889, 2018.

[9] B. Sahiner, A. Pezeshk, L. M. Hadjiiski et al., "Deep learning in medical imaging and radiation therapy," Medical Physics, vol. 46, no. 1, pp. e1-e36, 2019. 
[10] R. Feng, M. Badgeley, J. Mocco, and E. K. Oermann, “Deep learning guided stroke management: a review of clinical applications," Journal of Neurointerventional Surgery, vol. 10, no. 4, pp. 358-362, 2018.

[11] A. Hosny, C. Parmar, T. P. Coroller et al., "Deep learning for lung cancer prognostication: a retrospective multi-cohort radiomics study," PLoS Medicine, vol. 15, no. 11, Article ID e1002711, 2018.

[12] F. Liu, H. Jang, R. Kijowski, T. Bradshaw, and A. B. McMillan, "Deep learning MR imaging-based attenuation correction for PET/MR imaging," Radiology, vol. 286, no. 2, pp. 676-684, 2018.

[13] J. Wallner, M. Schwaiger, K. Hochegger, C. Gsaxner, W. Zemann, and J. Egger, "A review on multiplatform evaluations of semi-automatic open-source based image segmentation for cranio-maxillofacial surgery," Computer Methods and Programs in Biomedicine, vol. 182, Article ID 105102, 2019.

[14] B. Ibragimov and L. Xing, "Segmentation of organs-at-risks in head and neck CT images using convolutional neural networks," Medical Physics, vol. 44, no. 2, pp. 547-557, 2017.

[15] J. Schreier, A. Genghi, H. Laaksonen, T. Morgas, and B. Haas, "Clinical evaluation of a full-image deep segmentation algorithm for the male pelvis on cone-beam CT and CT," Radiotherapy \& Oncology, vol. 145, pp. 1-6, 2020.

[16] F. Tang, S. Liang, T. Zhong et al., "Postoperative glioma segmentation in CT image using deep feature fusion model guided by multi-sequence MRIs," European Radiology, vol. 30, no. 2, pp. 823-832, 2020.

[17] A. Horiuchi, T. Ikuse, and N. Tanaka, "Cold snare polypectomy: indications, devices, techniques, outcomes and future," Digestive Endoscopy, vol. 31, no. 4, pp. 372-377, 2019.

[18] M. Izzy, M. A. Virk, A. Saund, J. Tejada, F. Kargoli, and S. Anand, "Accuracy of endoscopists' estimate of polyp size: a continuous dilemma," World Journal of Gastrointestinal Endoscopy, vol. 7, no. 8, pp. 824-829, 2015.

[19] J.-S. Park, B. W. Bang, J. Shin et al., "A case of esophageal fibrovascular polyp that induced asphyxia during sleep," Clinical Endoscopy, vol. 47, no. 1, pp. 101-103, 2014.

[20] S. Din, A. Ball, S. Riley, P. Kitsanta, and S. Johal, "A randomized comparison of cold snare polypectomy versus a suction pseudopolyp technique," Endoscopy, vol. 47, no. 11, pp. 1005-1010, 2015. 